\title{
Application of Click Chemistry in the Development of Peptide Based HIV Fusion Inhibitors
}

\author{
Mukesh M Mudgal*1 and Nagaraju Birudukota² \\ ${ }^{1}$ Department of Chemistry and Biochemistry, Florida International University, 11200 SW $8^{\text {th }}$ street, Miami, Florida- 33199, USA
}

${ }^{2}$ Department of Chemistry and Physics, Halmos College of Natural Sciences and Oceanography, Nova Southeastern University, Fort Lauderdale, FL 33314, USA

Received: 制: August 16, 2018; Published: 制: November 05, 2018

*Corresponding author: Mukesh M Mudgal, Department of Chemistry and Biochemistry, USA

\begin{abstract}
Human Immunodeficiency Virus (HIV) continues to be a major global public health issue. Inhibition of HIV envelope fusion with the CD4 cell membrane prevents the entry of HIV into the CD4 cells providing a novel approach to the treatment of HIV infection. Thus, interference in the fusion of the virus with the co-receptor substrate appears to be a specific and potential way to fight HIV infection and replication. Applications of click chemistry are spreading in the field of drug discovery and it became a powerful tool for the synthesis of medicinally important compounds.

Remarkably, the $\mathrm{Cu}$ (I)-Catalyzed Azide-Alkyne Cycloaddition (CuAAC) click chemistry has become a superior approach for the synthesis of privileged medicinal skeletons in the discovery of anti-HIV agents. Click reactions got enormous popularity because of a high degree of reliability, complete specificity (chemoselectivity and regioselectivity) and it employs chemical reactions that are wide in scope, of high yielding and produce very little or no by-products. In this review, we outlined current approaches towards the development of peptide based HIV fusion inhibitors employing click chemistry.
\end{abstract}

\section{Introduction}

As per the statistics reported by HIV.gov, 36.7 million people worldwide were living with HIV/AIDS and 1.8 million people worldwide became newly infected with HIV in 2016, which averages about 5,000 new infections per day. About $70 \%$ of the people living with HIV around the world are aware of their HIV status while the other 30\% (estimated 11 million) people are not even aware of their HIV status. In 2016, 39,782 people were diagnosed with HIV in the U.S and more than 1.1 million people in the U.S. alone are living with HIV today. Men's who have sex with men are at higher risk of getting infected by HIV and represents an estimated 26,200 of these new HIV infections. Approximately 1 million people died from AIDSrelated illness since 2016 and around 35.0 million people have died since the start of the epidemic [1]. Research laboratories around the globe have been working to understand, diagnose, treat, and prevent HIV and HIV associated conditions [2-4].

Antiretroviral drugs used for the management of HIV/AIDS are categorized into six types depending on the stage of the viral life cycle where they are targeted. They are,

a) Nucleoside Reverse Transcriptase Inhibitors (NRTIs) and Nucleotide Reverse Transcriptase Inhibitors (NtRTIs) b) Non-Nucleoside Reverse Transcriptase Inhibitors (NNRTIs)

c) Protease inhibitors

d) Fusion inhibitors

e) Chemokine Receptor antagonists (CCR5 Antagonists) and

f) Integrase inhibitors.

Combination of antiretroviral drugs, referred as Highly Active Antiretroviral Therapy (HAART), which is considered as the most effective treatment model for AIDS. Protease inhibitors and Fusion inhibitors play a very important role in HAART $[5,6]$. Fusion inhibitors work by blocking the fusion of the HIV envelope with the CD4 cell membrane, thus preventing the entry of HIV into the CD4 cells, which is one of the important stages in the HIV life cycle. This interference of virus fusion with the co-receptor substrate appears to be specific and potentially the best way to interfere with HIV infection and replication. The three entry inhibitors that are currently available are Enfuvirtide-which prevents HIV from entering the CD4 T cell, Ibalizumab-blocks HIV from binding to the CD4 receptor and Maraviroc-blocks HIV from binding to a coreceptor [7]. 
Combination antiretroviral therapy has revolutionized the management of HIV infection but despite this success, antiretroviral regimens predictably fail in a large percentage of patients. Antiretroviral drugs also produce some side effects depending on the individual body type and the level of HIV infection. Therefore, there is a continuing research effort to discover, develop and deliver new antiretroviral drugs. HIV fusion inhibitors represent a novel class of antiretroviral drugs and the discovery of new, safe and potent fusion inhibitors are urgently needed.

Click chemistry has emerged as a powerful tool in drug discovery. The term "click chemistry reaction", defined by Nobel laureate KB Sharpless and associates in 2001, [8,9] served as a model reaction for the generation of novel pharmacophores. Compounds containing carbon-heteroatom-carbon bonds (mostly N, O, and S) can be efficiently synthesized by employing click chemistry in an aqueous environment [10-13] Click reactions got enormous popularity because it employs chemical reactions that are wide in scope, of high yielding and produce very little or no by-products. Click chemistry employs the copper (I) catalyzed 1,2,3- triazole formation from azides and terminal acetylenes to yield useful and versatile compounds. Triazole serves as an interesting class of molecule and an active pharmacophore in many biologically active compounds. It exists in two types, the 1,2,3-triazoles, and the 1,2,4-triazoles. Synthesis and biological applications of 1,2,3-triazoles and 1,2,4-triazoles have previously been extensively studied [14-16]. In this review, we would like to provide an overview of the application of click chemistry in the development of peptide based HIV Fusion inhibitors.

\section{Peptide based HIV Fusion Inhibitors}

$\mathrm{Cu}$ (1)-catalyzed click chemistry reaction is extensively used chemoselective reaction for drug discovery and investigation of biological function [6]. It is a method of choice for the synthesis of conjugates of small molecules, peptides and proteins [17]. T20 also known as Enfuvirtide is among the well-studied peptide fusion inhibitors and is the first member of the class approved by FDA for clinical use. T1249 and T2635 are other highly potent fusion inhibitors [18]. However, rapid protease degradation, drug resistance, and lengthy sequencing processes are the limitations associated with long-peptide inhibitors. To overcome these limitations, several homodimers, heterodimers of peptides, and non-peptide small molecule fusion inhibitors have been developed by employing click chemistry [19,20].

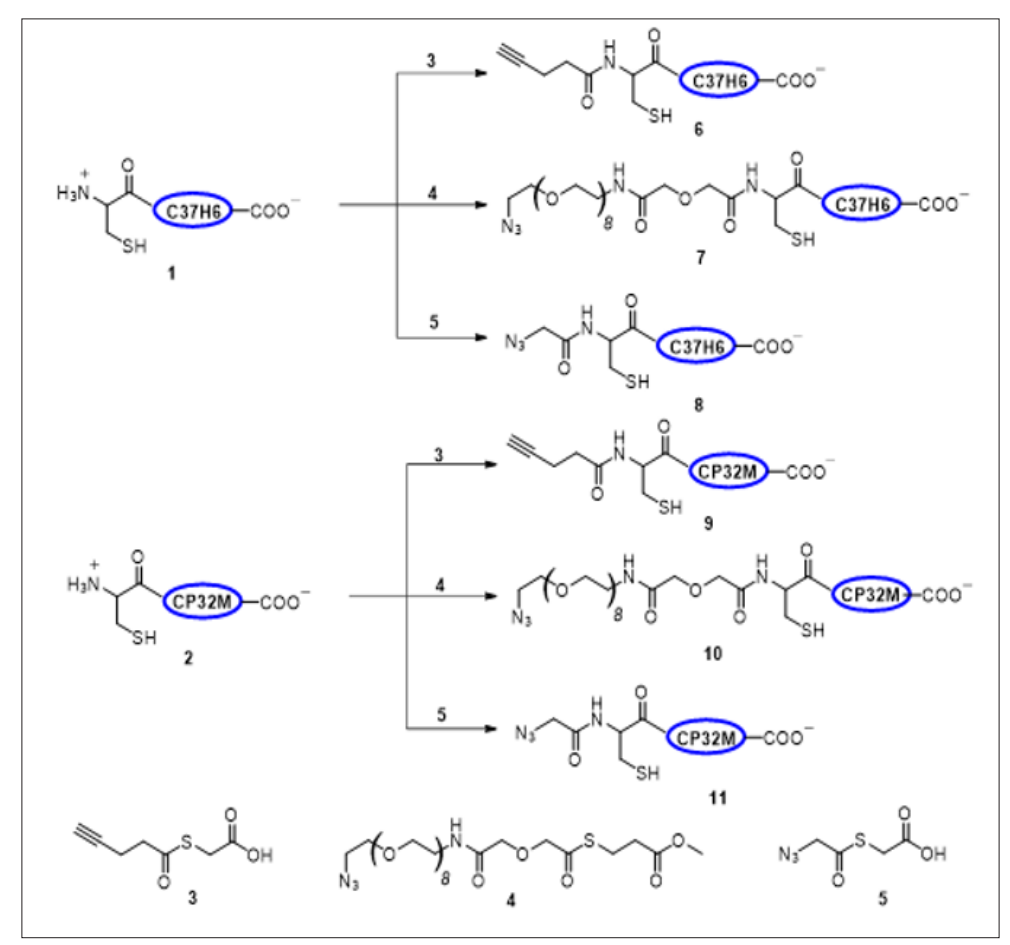

Figure 1: Synthesis of azide and alkyne moieties using NCL method.

In 2013, Xiao et al. reported a synthesis of homodimers and heterodimers of HIV fusion inhibitor peptides C37H6 and CP32M using a combination of native chemical ligation and CuAAC click chemistry approach [21]. Peptide inhibitors were first produced by recombinant protein expression and then the alkyne and azide groups were introduced into $\mathrm{C}_{37} \mathrm{H}_{6}$ and $\mathrm{CP} 32 \mathrm{M}$ by native chemical ligation method (Figure 1). The azide and alkyne groups are then subjected to CuAAC click chemistry employing $\mathrm{CuSO}_{4}$ and
L-ascorbic acid in denaturing buffer to form protein homodimers or heterodimers with variable linker lengths (Figure 2). Xiao and co-workers also performed the cell-cell fusion inhibition assay on the synthesized homodimers and heterodimers and found that the dimer obtained with a short linker did not demonstrate better antiviral activity compared to the monomers. Whereas the dimers with longer linker were four times more potent than the corresponding monomers. 


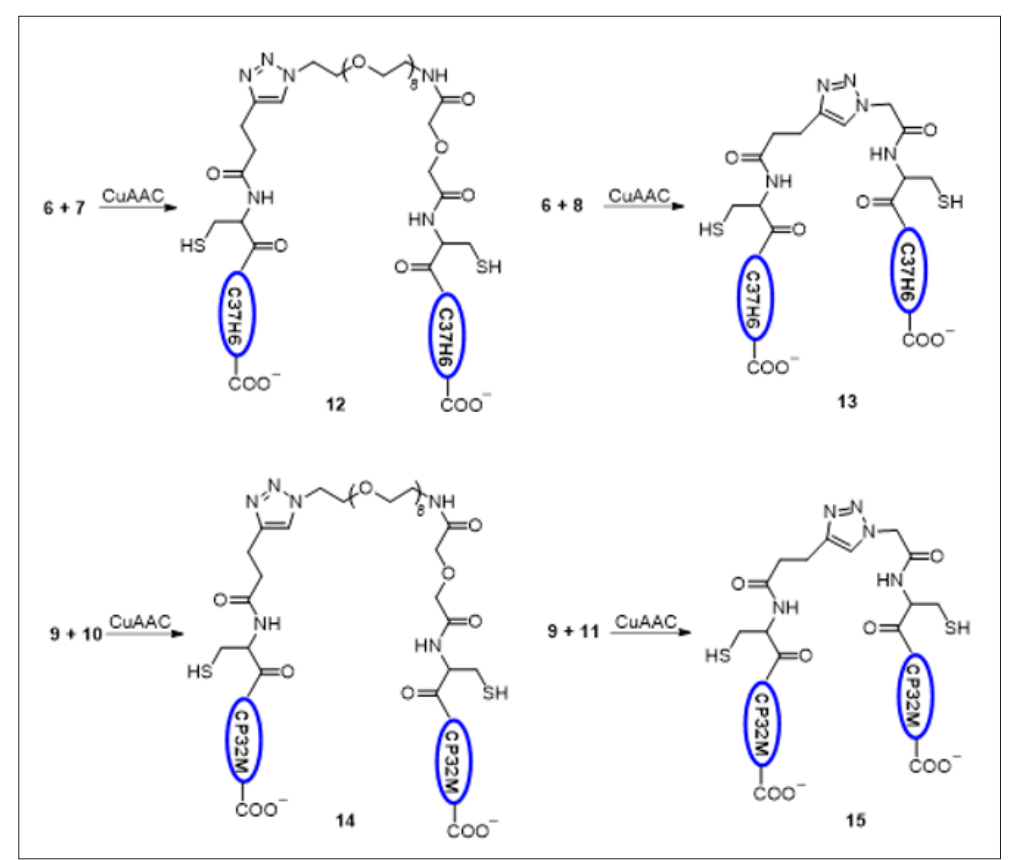

Figure 2: Synthesis of homo and heterodimers of fusion inhibitors using click chemistry.

Enfuvirtide has a lengthy sequence of 36 amino acids. Quick protease degradation and drug resistance are some of the problems reported for Enfuvirtide therapy which prompted the development of new generations of C-peptides. To overcome the problems associated with Enfuvirtide in 2016, Liang et al reported several nonpeptide small-molecule fusion inhibitors, which include phenylpyrroles, furan derivatives, and indole-based compounds. These inhibitors show a similar mechanism of action to C-peptides by targeting gp41 N-trimer. However, the main drawback of short peptides is that they adopt random confirmations and lose pharmacological usefulness. To overcome this shortcoming, Liang and co-worker's prepared conjugates of peptide and small molecules that target the gp41 with a synergistic effect. These conjugates showed promising anti-HIV-1 fusion activity, proposing a new method for the development of short-peptide HIV-1 fusion inhibitors.

To develop short peptides such as T26, T22, and T19, the peptide T2635 was considered as a lead compound due to its $\alpha$-helices stability in solution and reduced into short peptides by eliminating several inessential amino acid residues. The $\mathrm{N}$-terminus of these peptides are coupled with pocket-targeted small molecules through a $\beta$-alanine employing click chemistry. Indole derivative of (17) and the N-carboxyphenylpyrrole ligand GLS-22 derivative (19) were considered as small molecules due to their elongated molecule scaffolds and low micromolar EC50 anti-fusion potentials (Figure 3). Based on structure-activity relationship analysis, the methyl ester group on ring D of 16 and the carboxylic acid located at the para position of the phenyl ring of GLS-22 were substituted with propargyl ester. This substituted propargyl ester acts as a conjugated intermediate and linked to T2635-derived peptides through a triazole linkage by using click chemistry (Figure 4). The azide group needed for the conjugation was introduced at the N-terminus of the peptide through acylation with azido acetic acid. This purified azide-peptide coupled with the alkyne of small molecules via click chemistry (CuAAC) to produce T2635 derived peptides.

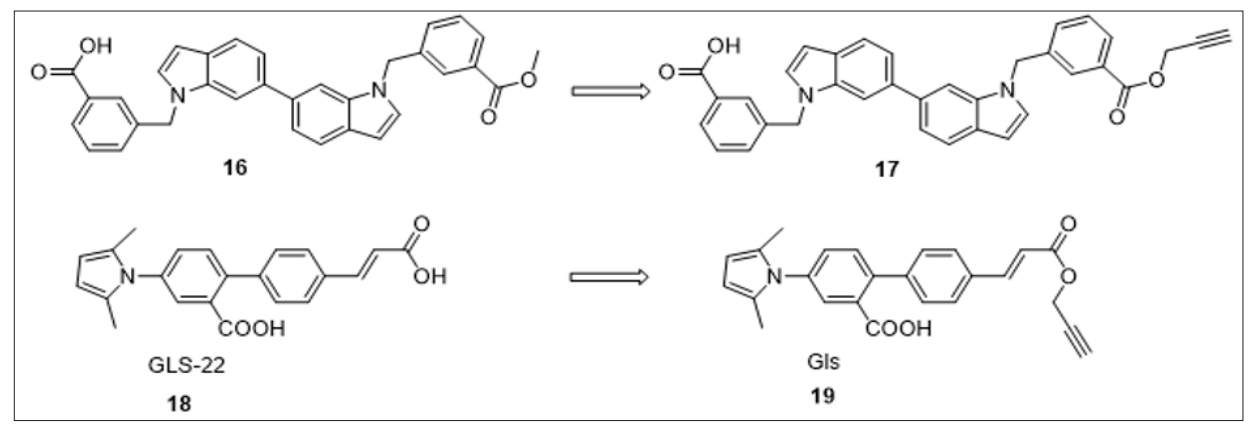

Figure 3: Structures of small molecule fusion inhibitors and their derivatives. 


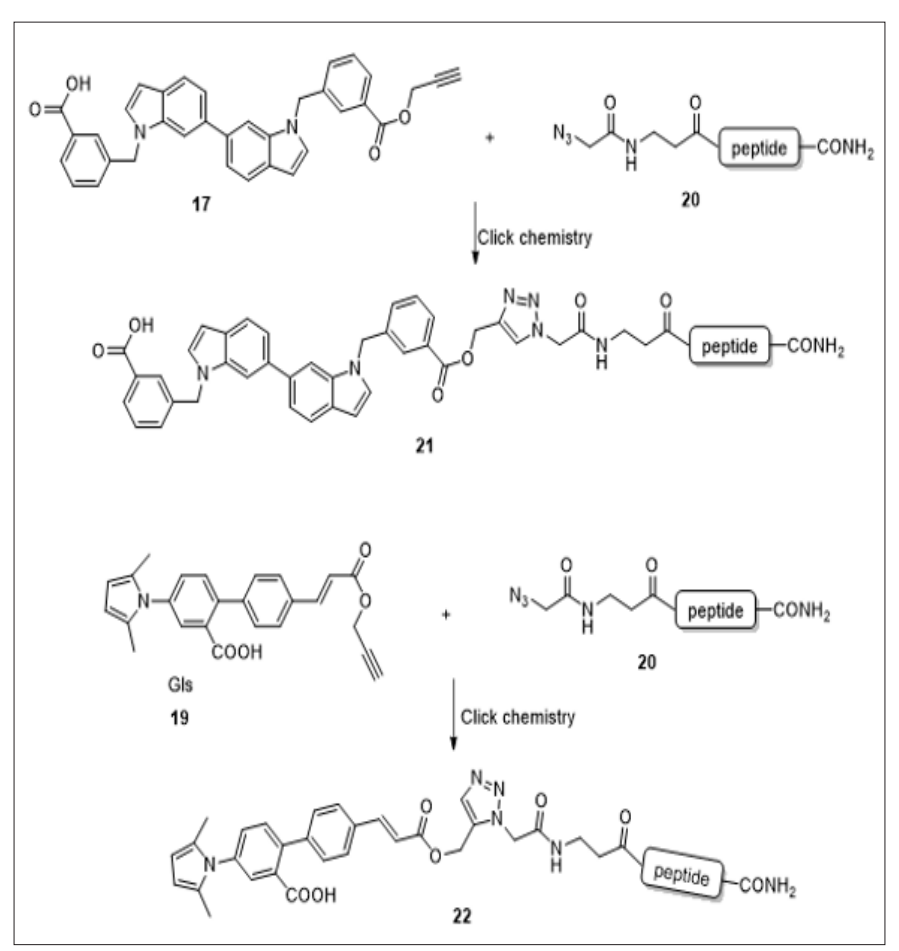

Figure 4: Synthesis of small molecule-peptide conjugate fusion inhibitors.

Peptide C34 that has a similar amino acid sequence as that of T20 has shown promising results in fusion inhibition. However, this compound is restricted to laboratory use due to its poor solubility in physiological conditions [22,23]. C34 and T20 both have Helix Zone-Binding Domain (HBD). Previous studies have shown that the HBD exhibited minimal anti-HIV-1 activity and the HBD peptides containing Pocket-Binding Domain (PBD) at N-terminus or a Lipid-Binding Domain (LBD) at C-terminus are more potent than HBD alone $[24,25]$. Wang et al. reported a design, synthesis and structure-activity relationship of novel HIV-1 fusion inhibitor hybrid molecules in which triterpene sapogenins were used to increase activity of HBD peptides [23]. They speculated that these bioactive natural saponins play an important role as PBD/LBD when added to the N/C -terminus of HBD peptide such as P26. Based on previous studies, triterpene saponins such as betulinic acid, oleanolic acid, and ursolic acid were selected for their synthetic study. The derivatives of these saponin acids containing terminal alkyne moiety were designed and synthesized (not shown here) as one of the intermediates in click coupling reaction with HBD derived P26 peptide (Figure 5). These new hybrid conjugate peptide molecules were tested against HIV-1 Env-mediated cellcell fusion and HIV-1IIIB (subtype B, X4) replication. Among all the conjugates, P26-BApc (28), showed promising anti-HIV-1 activity.

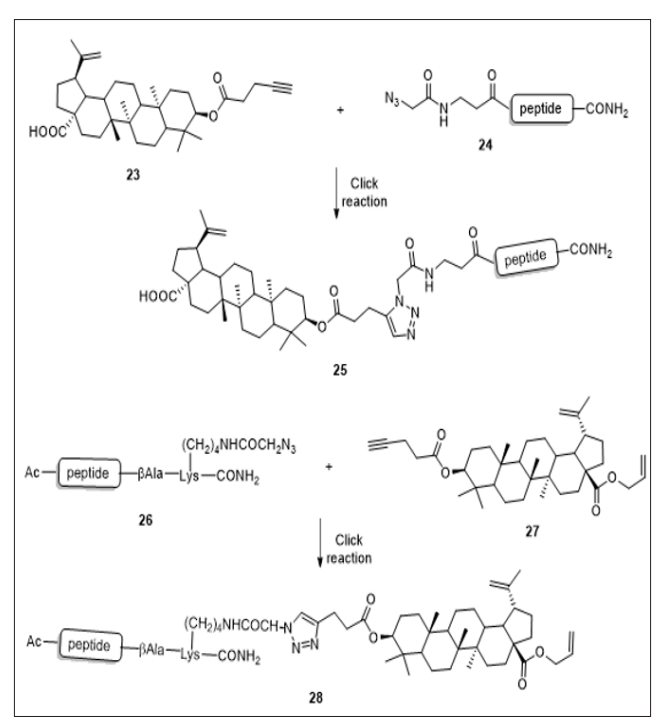

Figure 5: Synthetic method for preparing BAc-P26 (25) and P26-BApc (26). 


\section{Conclusion}

This review focuses on the application of click chemistry in the development of peptide based HIV fusion inhibitors. The applications of click chemistry are dispersing in the field of drug discovery, ranging from discovery through combinatorial chemistry to the field of proteomics employing the bioconjugation reactions. Click chemistry played an important role in the discovery of antiviral agents. This critical review summarizes the progress made so far in the discovery of peptide based HIV fusion inhibitors employing click chemistry approach. The click chemistry-based approach has become a popular protocol to synthesize HIV fusion inhibitors and it can be anticipated that in the future it will make further contributions to anti-HIV drug discovery. A lot of research is still needed in the area of antiretroviral drug expansion to discover new, safe, and potent fusion inhibitors that are less prone to the development of resistance.

\section{References}

1. US. Statistics.

2. Martinez SS, Sherman KE, Campa A, Rouster SD, Seminario L, et al. (2017) The interrelationships between PNPLA3 variants, BMI, liverrelated apoptosis and oxidative stress in HIV plus Adults from the (Miami Adults Studies in HIV) MASH cohort. Hepatology 66: 363A-363A.

3. Hernandez J, Mugdal M, Stewart T, Campa A, Martinez SS, et al. (2017) Effects of antioxidant supplementation on liver fibrosis in HIV+ individuals on Antiretroviral Therapy (ART) in the Miami Adult Studies in HIV (MASH) cohort. FASEB J. 31:1_supplement, 972.15-972.15.

4. Bertozzi S, Padian NS, Wegbreit J, DeMaria LM, Feldman B, et al. (2006) HIV/AIDS Prevention and Treatment. World Bank, Chapter 18.

5. Midde NM, Patters BJ, Rao PS, Cory TJ, Kumar S (2016) Investigational protease inhibitors as antiretroviral therapies. Expert Opin Investig Drugs 25: 1189-1200.

6. Mudgal MM, Birudukota N, Doke MA (2018) Applications of click chemistry in the development of HIV protease inhibitors. Inter J Med Chem 2018: 9.

7. U.S. Department of Veterans Affairs. Entry inhibitors - HIV/AIDS.

8. Kolb HC, Finn MG, Sharpless KB (2001) Click chemistry: Diverse chemical function from a few good reactions. Angew Chem Int Ed Engl 40: 2004-2021.

9. Wu P, Feldman AK, Nugent AK, Hawker CJ, Scheel A, et al. (2004) Efficiency and fidelity in a click-chemistry route to triazole dendrimers by the copper(I)-catalyzed ligation of azides and alkynes. Angew Chem Int Ed Engl 43: 3928-3932.

10. Riddler SA, Haubrich R, DiRienzo AG, Peeples L, Powderly WG, et al. (2008) Class-sparing regimens for initial treatment of HIV-1 infection. $\mathrm{N}$ Engl J Med 358: 2095-2106.

\section{ISSN: 2574-1241}

DOI: 10.26717/BJSTR.2018.10.002004

Mukesh M Mudgal. Biomed J Sci \& Tech Res

This work is licensed under Creative

Commons Attribution 4.0 License

Submission Link: https://biomedres.us/submit-manuscript.php
11. Wang JH, Pan CW, Li YT, Meng FF, Zhou HG, et al. (2013) Safe and highly efficient syntheses of triazole drugs using $\mathrm{Cu}_{2} \mathrm{O}$ nanoparticle in aqueous solutions. Tetrahedron Letters 54: 3406-3409.

12. Moses JE, Moorhouse AD (2007) The growing applications of click chemistry. Chem Soc Rev 36: 1249-1262.

13. Kolb HC, Sharpless KB (2003) The growing impact of click chemistry on drug discovery. Drug Discov Today 8: 1128-1137.

14. Mudgal MM, Singla RK, Bhat VG, Shenoy GG (2009) Synthesis and antimicrobial activity of some new [1,2,4] triazolo[3,4-B][1,3,4] thiadiazol derivatives. Pharmacologyonline 188-193.

15. Gonzaga DT, da Rocha DR, da Silva Fde C, Ferreira VF (2013) Recent advances in the synthesis of new antimycobacterial agents based on the 1H-1,2,3-triazoles. Curr Top Med Chem 13: 2850-2865.

16. Mansoory JH, Rajput SS (2015) Synthesis, reactivity and biological evaluation of triazole: Recent developments. Int J Pharmacy Pharmaceut Sci 7: 20-32.

17. Weikart ND, Sommer S, Mootz HD (2012) Click synthesis of ubiquitin dimer analogs to interrogate linkage-specific UBA domain binding. Chem Commun (Camb) 48: 296-298.

18. Berkhout B, Eggink D, Langedijk H, Sanders RW (2010) Towards improved anti-HIV peptides that prevent viral escape. Pept Sci 43: 20112013.

19. Jiang S, Zhao Q, Debnath AK (2002) Peptide and non-peptide HIV fusion inhibitors. Curr Pharm Des 8: 563-580.

20. Liang G, Wang H, Chong H, Cheng S, Jiang X, et al. (2016) An effective conjugation strategy for designing short peptide-based HIV-1 fusion inhibitors. Org Biomol Chem 14: 7875-7882.

21.Xiao J, Tolbert TJ (2013) Modular assembly of dimeric HIV fusion inhibitor peptides with enhanced antiviral potency. Bioorg Med Chem Lett 23: 6046-6051.

22. Cai L1, Jiang S (2010) Development of peptide and small-molecule HIV-1 fusion inhibitors that target gp41. Chem MedChem 5: 1813-1824.

23. Wang $\mathrm{C}$, Lu L, Na H, Li X, Wang $\mathrm{Q}$ et al. (2014) Conjugation of a nonspecific antiviral sapogenin with a specific HIV fusion inhibitor: A promising strategy for discovering new antiviral therapeutics. J Med Chem 57: 7342-7354.

24.Zhi Qi, Weiguo Shi, Na Xue, Chungen Pan, Weiguo Jing, et al. (2008) Rationally designed anti-HIV peptides containing multifunctional domains as molecule probes for studying the mechanisms of action of the first and second generation HIV fusion inhibitors. J Biol Chem 283: 30376-30384.

25. Liu S, Jing W, Cheung B, Lu H, Sun J, et al. (2007) HIV gp41 C-terminal heptad repeat contains multifunctional domains: Relation to mechanisms of action of anti-HIV peptides. J Biol Chem 282: 9612-9620.

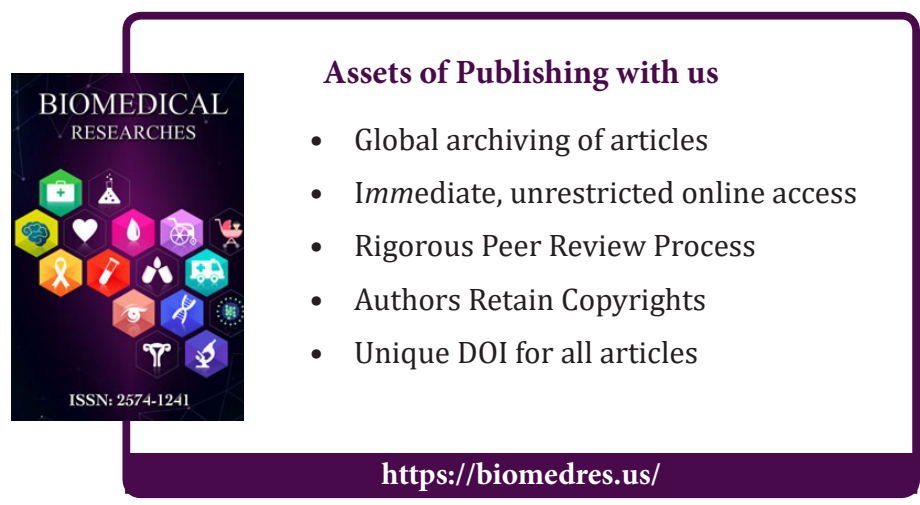

